\title{
Reconstrucción del tejido social en el ámbito escolar: una propuesta desde trabajo social
}

\section{Reconstruction of the social fabric in the school environment: a proposal from social work}

\author{
Ornelas, Adriana, M.Sc. Tello, Nelia, M.Sc.
}

Universidad Nacional Autónoma de México - UNAM. Escuela Nacional de Trabajo Social. México. Correspondencia: adrianao2000@yahoo.com, neliatello@me.com

\section{RESUMEN}

La actual descomposición social se caracteriza por el individualismo, la indiferencia y la falta de solidaridad que provocan el deterioro del tejido social. La violencia es una de las formas de relación social dominante que se ha normalizado en nuestras sociedades y ha permeado las instituciones escolares. En este sentido, interesa caracterizar los procesos sociales que se generan en el ámbito escolar, en donde se reproduce y recrea la violencia, la exclusión, el rechazo, la desconfianza, enfatizando que la escuela no solo es una "caja de resonancia" de lo que sucede a nivel societal, sino que también representa una posibilidad para incidir en la modificación de las formas relacionales imperantes y promover procesos de inclusión, aceptación y confianza entre los diferentes actores de la comunidad escolar, por lo que se presentará un modelo de intervención diseñado desde Trabajo Social que pretende contribuir -desde la especificidad de esta disciplina- a la construcción de una sociedad diferente, incluyente, democrática.

Palabras clave: Tejido social, intervención de trabajo social, ámbito escolar, inclusión social. 
Ornelas y Tello - Reconstrucción del tejido social en el ámbito escolar

\begin{abstract}
The current social decomposition is characterized by individualism, indifference and lack of solidarity that cause the deterioration of the social fabric. Violence is one of the forms of dominant social relation that has normalized in our societies and has permeated school institutions. In this sense, it is interesting to characterize the social processes that are generated in the school environment, where violence, exclusion, rejection and distrust are reproduced and recreated, emphasizing that school is not only a "sounding board" of which happens at a societal level, but it also represents a possibility to influence the modification of the prevailing relational forms and promote processes of inclusion, acceptance and trust, among the different actors of the school community, so that a model of intervention designed from Social Work which aims to contribute -from the specificity of this discipline- to the construction of a different, inclusive, democratic society.
\end{abstract}

Keywords: Social fabric, social work intervention, school environment, social inclusion.

\section{Lo social desde la perspectiva de trabajo social}

Es en los últimos años cuando con frecuencia se encuentran alusiones al tejido social y a la cohesión social como aspectos a analizar para mejorar la vida en sociedad. En este contexto, se considera que el trabajo social contemporáneo está Ilamado a cumplir con una función fundamental: la de intervenir en los problemas que se generan en los procesos y relaciones sociales y que impactan en el deterioro, fragmentación y ruptura del tejido social, asumiendo, como señala Tello (2015) que "el trabajo social es una profesión que interviene en situacionesproblema, desde lo social y en lo social, generando cambios racionales e intencionales concretos, en el aquí y ahora de los sujetos sociales". Por ello, para nosotras centrar la especificidad disciplinar en lo social, significa focalizar la intervención en los vínculos, en las relaciones e interacciones sociales, en los procesos relacionales conflictivos como son la violencia, discriminación, abuso, rechazo, subordinación, dependencia, exclusión, competencia, dominación, desconfianza, entre otros, para generar procesos de aceptación, confianza, inclusión, solidaridad, etc., tendientes a la reconstrucción del tejido social.
Para comenzar, se hará una breve exploración del concepto tejido social, desagregando sus elementos constitutivos: la palabra tejido, alude a la idea de unidades independientes que se juntan para formar algo, ese algo sostiene, contiene y toma una nueva forma colectiva; es decir, es lo que resulta de entrelazar. El término social hace referencia al conjunto de individuos que conforman la sociedad. Para Falla y Chávez (2004) el tejido social se concibe como "un conjunto de interdependencias entre partes, elementos, procesos, donde se da una serie de relaciones internas e interdependientes que sirven de soporte emocional, cultural, físico, social y aún económico a sus interactuantes" (p.9). Castro y Gachón (2001) lo definen como "El entramado de relaciones cotidianas que implican a su vez relaciones de micro-vínculos en un espacio local y social determinado como lo es el barrio". Habermas (2000) señala que se trata de "un conjunto de relaciones efectivas que determinan las formas particulares de ser, producir, interactuar y proyectarse en los ámbitos familiar, comunitario, laboral y ciudadano" (p.65). Con base en ello, desde el trabajo social se entenderá al tejido social como el entramado de relaciones que establecen los sujetos sociales, en un espacio social determinado para, de 
manera organizada, obtener un fin o cumplir una función social.

Los sujetos son parte de diferentes formas organizativas que crean o a las que se integran y van perteneciendo; este entramado, en su conjunto, es lo que conforma el denominado tejido social. Sin embargo, diversas circunstancias van afectando dichas formas organizativas y es cuando se alude a los términos debilitamiento o ruptura del tejido social, provocando la actual descomposición social que se caracteriza por el individualismo, la competencia, la desconfianza, la indiferencia y la falta de solidaridad: "En la sociedad moderna, la intensidad de la división social del trabajo hace que la solidaridad sea más débil, que corresponda a intereses más particulares, donde la comunidad se diluye de diversas maneras" (Mota y Sandoval, 2011, p. 34 -35). En este contexto, la sensación de incertidumbre e indefensión se va agudizando, llevando a los sujetos a pensar en el otro como extranjero, como rival al que hay que temerle o, peor aún, como el que no es adecuado para establecer lazos amistosos o solidarios, provocándose la atomización de los sujetos y el consecuente resquebrajamiento de lo social.

Ante ello, es preciso ampliar la mirada y pensar en los procesos que dan origen a la fragmentación de los colectivos sociales, por ejemplo, habría que hablar de la discriminación, el rechazo, la indiferencia, la desconfianza, el aislamiento, que además provocan la pérdida del sentido de responsabilidad por el otro, la pérdida de cohesión, la falta de sentido colectivo y del bien común. Lo anterior implicaría el reconocimiento de la importancia de la dimensión social en las situaciones-problema que afectan procesos de organización y participación en escenarios colectivos; al brindar otra mirada, se abriría la posibilidad de buscar caminos alternativos para su solución. Como señala Bauman (2005:12): "Ios miedos actuales nacieron al brotar simultáneamente la liberalización y el individualismo, cuando ya se habían aflojado o roto los lazos de parentesco y vecindad que unían con firmeza a comunidades y corporaciones, lazos que se tenían por eternos o que al menos existían desde tiempos inmemoriales", de ahí la importancia de la intervención profesional en los procesos que afectan el tejido social.

\section{Caracterización del tejido social en las escuelas}

La familia, la escuela, el trabajo, el vecindario, son entornos sociales que favorecen la interacción entre los sujetos, pues gran parte de la vida cotidiana transcurre en estos $y$ ello significa una constante vinculación de unos con otros; para fines del presente texto, se centrará la atención en solo uno de estos: la escuela. Se comienza por reconocer que a esta se le han asignado dos funciones principales: la formación en determinadas áreas del conocimiento a través de la transmisión de contenidos y la socialización o formación social, que es la que habilita a los infantes y jóvenes estudiantes para entablar relaciones con los demás. Sin embargo, es preciso reconocer que esta última función se ha ido desdibujando y ha perdido relevancia; con ello no se afirma que no suceda, sino que en muy pocas ocasiones se trata de una acción intencional y direccionada; más bien forma parte del currículo oculto en donde más que los contenidos o discursos, son las acciones las que se están reproduciendo y aprendiendo: "Por su parte, las escuelas siguen cumpliendo su función socializadora....[pero] (sic)... será una socialización que reproducirá patrones de conducta de sobrevivencia en una sociedad en descomposición, y no el deber ser estipulado en el pacto de lo formal, que como sistema único dejó de operar, hace algunos años, en nuestra sociedad" (Tello, 2015, p.13). Así, la escuela, al ser una institución jerárquica, reproduce relaciones desiguales, casi siempre de dominio- 
sumisión, en donde lo que priva es el control, no solo administrativo, sino también social.

Comprender lo que sucede en el entramado social de la escuela, es decir, en la convivencia entre los diferentes actores, suele no ser un tema de interés, pues se prioriza la forma en la que se transmiten los conocimientos y su aprehensión por parte de los estudiantes: "Es una pena que el carácter socializante de la escuela...sea desatendido. Se habla casi en forma exclusiva de la enseñanza de los contenidos" (Freire, 2012, 44), sin tener en cuenta que, como se dijo antes, la escuela es un microcosmos que reproduce las relaciones sociales imperantes y por lo tanto se reproducen las manifestaciones de la ruptura del lazo social. En tiempos recientes es cuando se ha decidido retomar esta dimensión, sin embargo, solo se le considera como un 'contenido' que se aprende con un curso; se le dedican las horas 'muertas' o 'libres'; se asume como algo secundario y poco importante y por lo tanto, no se desarrolla como un proceso que se vive en lo cotidiano, no se pone en práctica ni se relaciona con el resto de lo que se enseña en la escuela.

Si bien se coincide con autores (Del Tronco, 2015; Ampudia, et.al., 2016) que afirman que la escuela es una caja de resonancia de lo que sucede en la sociedad en general, parece pertinente hacer un matiz y señalar que si bien la escuela es reproductora de la sociedad y por lo tanto de las formas relacionales dominantes, también es un espacio de re-creación de dicha sociedad y de sus interacciones, por lo que existe la posibilidad de generar un aprendizaje alternativo de relaciones y habilidades sociales para la convivencia social; de aprender a ser y estar con el otro.

Como se sabe, en la escuela las interacciones son constantes y cotidianas entre los diferentes actores: entre estudiantes; entre estudiantes y docentes; entre estudiantes y autoridades; entre docentes; entre docentes y autoridades; entre autoridades; entre padres de familia; entre padres y estudiantes; entre padres y autoridades y entre padres y docentes; tejiéndose con ello un entramado relacional al que se denomina tejido socio-escolar, entendiéndolo entonces

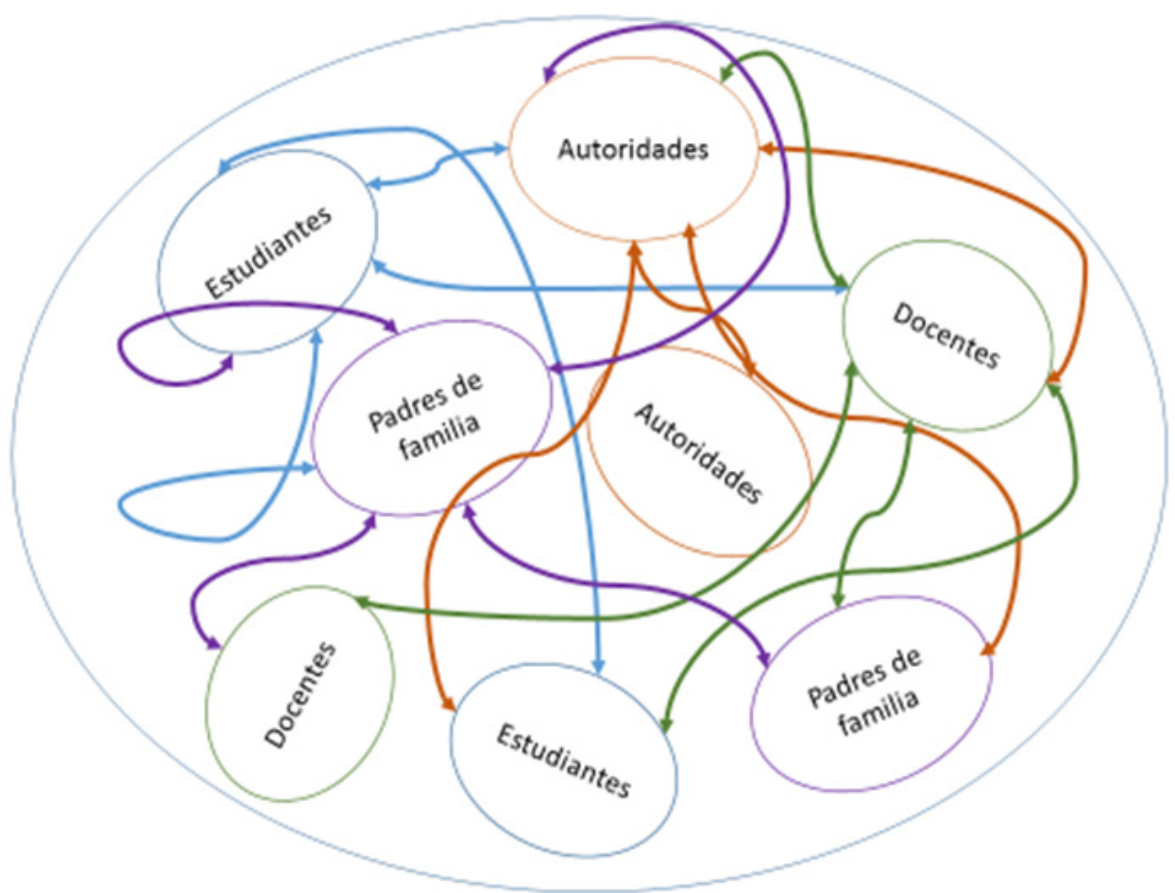

Figura1. Entramado relacional en la escuela 
como el conjunto de relaciones que se dan en la interacción entre los diferentes actores de la comunidad escolar, que está determinado por las normas, los valores, los canales de comunicación y vínculos que se generan entre ellos.

Diversos autores se han referido al desgaste que puede experimentar el tejido social y le han denominado de diferentes maneras, siendo las formas más comunes de nombrarlo como fragmentación o ruptura, que provoca distanciamientos y polarizaciones, que dificultan las interacciones entre los sujetos; por supuesto, estas situaciones se presentan en el entramado relacional de las escuelas, en donde, desde nuestra perspectiva, se puede manifestar en tres niveles:

Deterioro: Serefierea su debilitamiento, al desgaste cotidiano de las relaciones que casi siempre resulta imperceptible y por lo tanto no se atiende, se deja pasar y al agudizarse deriva en la fragmentación.

Fragmentación: Se presenta cuando se provoca una escisión que divide al colectivo, que lo separa en sub-grupos que no establecen relaciones con otros sub-grupos. De igual forma, su polarización puede conducir a la ruptura.

Ruptura: Se origina cuando las diferencias y oposiciones hacen imposible la convivencia con los otros - sean considerados semejantes 0 diferentes-; es cuando se interrumpen o suspenden las relaciones con los otros.

A continuación, se explicitan algunas relaciones sociales que se generan y/o reproducen en la escuela desde el abordaje del trabajo social, para con base en ello proponer una estrategia de intervención en lo social. Entre los procesos sociales conflictivos que se dan en las escuelas se encuentran: simulación, complicidad, competencia, discriminación, descalificación, estigmatización, indiferencia, desconfianza, aislamiento, segregación, que pro- vocan el aislamiento y la exclusión; bajo el entendido de que todos estos procesos se desarrollan de manera entretejida en las diferentes interacciones entre sujetos y actores sociales.

Simulación: significa darle al otro lo que pide para no tener conflictos: "La simulación es el valor asumido en la escuela de manera genérica por todos sus actores; nadie tendrá el apoyo -ni la directora, ni los maestros, ni los alumnos- de la norma o de la institución para develar algo que implique denuncia, que tenga que ver con la autoridad, con la aceptación del problema, con su desmenuzamiento y su atención" (Tello, 2015, p. 33); y así lo manifiestan algunos estudiantes: "Le doy lo que pida (la maestra) para que esté contenta y no me diga nada" (GF1. En: Ornelas, 2013, p.103).

Complicidad: es parte de los "pactos" que se establecen entre los actores de la comunidad escolar y se refiere a aquellas situaciones en las que una o varias partes no cumplen con lo que se espera de ellos, pero dicho incumplimiento es aceptado, tolerado y silenciado, con lo que se perpetúan situaciones que tendrían que modificarse. Un ejemplo de ello es en el trabajo en equipos que realizan los estudiantes: "Si los otros no hacen su parte del trabajo, aun así los ponemos en los trabajos, pues no sabemos decir que no y además pensamos: me va a dejar de hablar, me van a ver como la payasa y ya no me van a aceptar" (GF2. En: Ornelas, 2013, p.106).

Competencia: se manifiesta en el individualismo de los actores, sean estudiantes, padres o docentes; la gran mayoría manifiesta su preferencia por el trabajo individual pues, en su experiencia, no se aprecia el beneficio del trabajo en conjunto y sí en cambio, este ha sido percibido como una 'carga'; por lo tanto, lo que buscan es 'salvarse' a sí mismos, sin considerar que "En cuanto la competencia sustituye la solidaridad, las personas se ven abandonadas a sus 
propios recursos, penosamente escasos y obviamente insuficientes" (Bauman, 2005, p.14).

Discriminación: se refiere a la tendencia a dar un trato desfavorable a todo aquel que se considera diferente, lo que va provocando la atomización del colectivo en subgrupos cada vez más pequeños que solo aceptan a los que consideran 'iguales'. Esta discriminación se da por factores diversos como la apariencia, la obesidad y el sobrepeso, la preferencia sexual, entre los más frecuentes.

Descalificación: el punto de vista divergente del propio es motivo de pleito, no de debate; de descalificación, no de argumentación; existe una invalidación de la palabra del otro a quien no se reconoce como 'digno' para opinar; se refiere también al descrédito del otro, por considerar que no cuenta con los elementos 'suficientes' para interactuar con alguien, lo cual se observa frecuentemente en las aulas cuando los estudiantes solo atienden a la palabra del profesor y no a la de sus compañeros.

Estigmatización: se establecen relaciones a través de etiquetas que cosifican a los sujetos, asignándoles un rol o papel que se considera inalterable y por lo tanto, las relaciones se dan desde lo pre-escrito, como lo expresan algunos estudiantes: "Siempre los docentes se fijan en los estudiantes que se sientan adelante y hacen la relación: adelante-listos; atrás-latosos y se aprende solo los nombres de estos dos grupos" (GF3.). "Los maestros deberían ser más objetivos, pues se crean una imagen del estudiante, los clasifican y ya no sale de eso y además lo hacen obvio" (GF3. En: Ornelas, 2013, p. 92).

Indiferencia: es una de las principales características de la vida moderna y se refiere a la falta de sentido de pertenencia $y$, por lo tanto, al desinterés en reconocer el vínculo de unos con otros; considerándose que los problemas son ajenos: "los maestros perciben los problemas, perciben a las víctimas de agresión, perciben a los que se drogan y son contadas las ocasiones en las que intervienen, prefieren no meterse en líos, saben que no serán apoyados por las autoridades y que los riesgos que corren son mayores a lo que puedan solucionar" (Tello, 2015, p. 18). En esta relación es frecuente la existencia del silencio, que nos interesa destacar, pues la ausencia de la palabra es un importante indicador de los conflictos en las relaciones entre los actores de la comunidad escolar; por un lado, puede estar indicando una manifestación de inconformidad ante una situación que no se hace explícita dado el autoritarismo existente; y también puede interpretarse como una expresión de la indiferencia por lo que acontece en la escuela, dejando así de involucrarse en cualquier asunto que suceda en esta.

Desconfianza: esta forma de relación lleva a mirar al otro como sospechoso, como ajeno, como no perteneciente, e incluso, como enemigo o rival, lo cual redunda en la dificultad de establecer relaciones con los demás. Así por ejemplo, ante un suceso de robo de un celular, los estudiantes y docente no vuelven a dejar sus mochilas y bolsas en el aula, se les ve con ellas todo el tiempo, se genera el rumor y la sospecha sobre el otro; entonces en este espacio social también "predomina la desconfianza en los demás y en sus intenciones, así como una actitud que niega o considera imposible tener fe en la constancia y en la fiabilidad del compañerismo humano" (Bauman, 2005: 9).

Segregación: se da con la conformación de subgrupos que establecen rivalidades entre sí y lo que prima son los intereses particulares, no el colectivo: "En los salones de clases se conforman los grupos excluyendo a algunos estudiantes que no demostraron capacidad de integración y que, por alguna razón ya sea física, intelectual o emocionallos chavos deciden segregarlos" (Tello, 
2015, p.60). Bauman (2005:57) se refiere a esta como "La tendencia a abandonar los lugares públicos y recluirse en islas de iguales es lo que termina siendo el principal impedimento para convivir con la diferencia, puesto que hace languidecer hasta suprimirla, la capacidad para el diálogo y la negociación" y con ello viene el aislamiento y la exclusión.

Todos estos procesos relacionales se ven reforzados por una estructura organizativa autoritaria en la que la impunidad y la violencia son las constantes que se reproducen en cada uno de sus actores: de funcionarios a profesores, de profesores a estudiantes; de estudiantes de tercero a los de segundo y de estos a los de primero, por citar algunos ejemplos. El control es el interés mayor y se estima a la convivencia como su contrario: "Tenemos escuelas sobre-normadas, hay un código...que tipifica actitud por actitud, práctica por práctica, para ser castigado". (Pérez, $2015,14)$. Así, se van desgastando las relaciones, aparece la sospecha sobre los otros a quienes se llega a concebir como enemigos. Se instala una resistencia a entablar relaciones sociales amplias y diversas; se prefiere formar grupos pequeños y cerrados y con ello se pierde el sentido de comunidad, de colectivo; se reducen los espacios de convivencia, hay un distanciamiento físico y social; se fragmentan las relaciones de reciprocidad, cooperación, corresponsabilidad, con la consecuente atomización de los sujetos en individuos aislados y entonces, se va generando la afectación del lazo social, en cualquiera de sus niveles, dentro y fuera de la escuela.

Ante ello, resulta indispensable modificar las formas relacionales en las escuelas, pues si pretendemos consolidar una sociedad democrática, son sus instituciones las que tienen que contribuir en ello, pues de lo contrario: "Me vuelvo tan falso como quien pretende estimular el clima democrático por medios y caminos autoritarios" (Freire,
$2012,48)$. En la escuela, cualquiera que sea el nivel de estudios, se pasa gran parte del tiempo de vida cotidiana, por lo tanto, es un espacio donde se establecen variedad de relaciones y se aprende de los estudiantes, docentes y autoridades acerca de cómo es la vida 'afuera'; de ahí la importancia de los procesos relacionales que se crean y recrean en la institución escolar.

\section{Propuesta de intervención: Construcción de Comunidades Escolares A.C.I.}

Se comienza por reconocer que la tendencia de la intervención del trabajo social en las escuelas ha sido:

1. Como coadyuvante de los profesores, directivos y administrativos, al dedicarse a pasar lista, registrar asistencias e inasistencias, control, emisión de reportes, vigilancia del cumplimiento del reglamento, etc.

2. A través del trabajo individualizado, es decir, atendiendo casos 'problema' para re-adaptarlos.

3. Realizando una serie de actividades inconexas entresícomo pláticas, periódicos murales, entrevistas individuales que no se direccionan en un mismo sentido ni con un fin común predeterminado.

Teniendo como principal propósito la homogeneización de los estudiantes para lograr el ideal del alumno: callado y obediente, que no pregunta, solo escucha.

A continuación, se presenta una propuesta diferente para reconstruir el tejido social en las escuelas, a la que se le ha denominado "Comunidades Escolares ACI", cuyo objetivo general es la construcción de convivencia solidaria en las comunidades escolares y sus entornos, a través de desencadenar procesos de aceptación, confianza e inclusión entre los jóvenes estudiantes y los distintos actores que los conforman, para fortalecer la cohesión entre ellos. 


\section{a) Fundamentos}

Esta propuesta para la intervención del trabajo social en las escuelas, postula el abordaje de la situaciónproblema desde la complejidad, lo cual significa romper con la lógica lineal causa-efecto, al comprender que los problemas sociales que se presentan en las escuelas se entretejen entre todos los actores, surgen de las formas en las que estos se relacionan $y$, por lo tanto, las posibilidades para su modificación radican en la capacidad de cambiar las formas relacionales imperantes. Para ello, es preciso tener una visión integral de la situación-problema a intervenir, para modificarla y no centrarse solo en uno de sus componentes o actores (p.e. las formas de relacionarse que tienen los jóvenes), pues ello conduce a intervenciones parciales, que no logran la modificación del problema.

La propuesta que a continuación se detalla, contrariaa lasmedidascoercitivas y de individualización del problema, es una apuesta por la alteridad, por la construcción del nosotros, en donde se asume la responsabilidad compartida $y$, por lo tanto, todos los actores tienen algo que cambiar. Se propone lograr la autorregulación de los sujetos a través de desarrollar habilidades sociales para la convivencia solidaria, en lugar de su aislamiento y estigmatización que solo genera mayores problemas de convivencia. Tiene su fundamento en la otredad, que implica el reconocimiento del otro como un yo y la aceptación de la diferencia como elemento que enriquece al colectivo y conforma el nosotros.

En el marco de esta propuesta, se entiende a la aceptación, la confianza y la inclusión (ACI) de la siguiente manera:

La aceptación es el respeto y el reconocimiento del sí y del otro, sin importar las diferencias o discrepancias que existan; la capacidad de vincularse y relacionarse con la diversidad. No es un acto individual, aislado; al contrario, tiene que ver con los demás, con la alteridad, con la construcción de un nosotros.

La confianza nos lleva a esperar, con cierta seguridad, una respuesta específica en nosotros mismos y en los otros. Alude también al ánimo y a la decisión para obrar, así como a la familiaridad en el trato. La confianza reduce la incertidumbre y permite al otro elegir con mayor manejo de la situación.

La inclusión es un proceso de acercamiento con el colectivo, significa pertenencia, trato igualitario, aceptación, reconocimiento. La inclusión genera lazos y vínculos relacionales entre los sujetos de la comunidad escolar, quienes se asumen como un colectivo responsable de su propia historia (Tello y Cruz, 2015).

Como se dijo antes, el objetivo de esta propuesta de intervención es la construcción de convivencia solidaria en las comunidades escolares y sus entornos, a través de desencadenar procesos de aceptación, confianza e inclusión entre los jóvenes estudiantes y los distintos actores, fortaleciendo la cohesión, destacando que si bien los jóvenes estudiantes son la unidad de intervención, al tratarse de procesos relacionales, se trabaja con todos los actores de la comunidad escolar, es decir, con un abordaje desde la complejidad, en la que se incluyen autoridades, funcionarios, maestros, padres de familia y vecinos del entorno.

\section{b) Propuesta metodológica}

La propuesta se compone de cuatro ejes y cuatro acciones transversales:

Ejes:

1. El fortalecimiento institucional, consiste en organizar grupos de análisis con los directivos y funcionarios de las escuelas, para re-conocer la situación de violencia que ocurre en la institución 


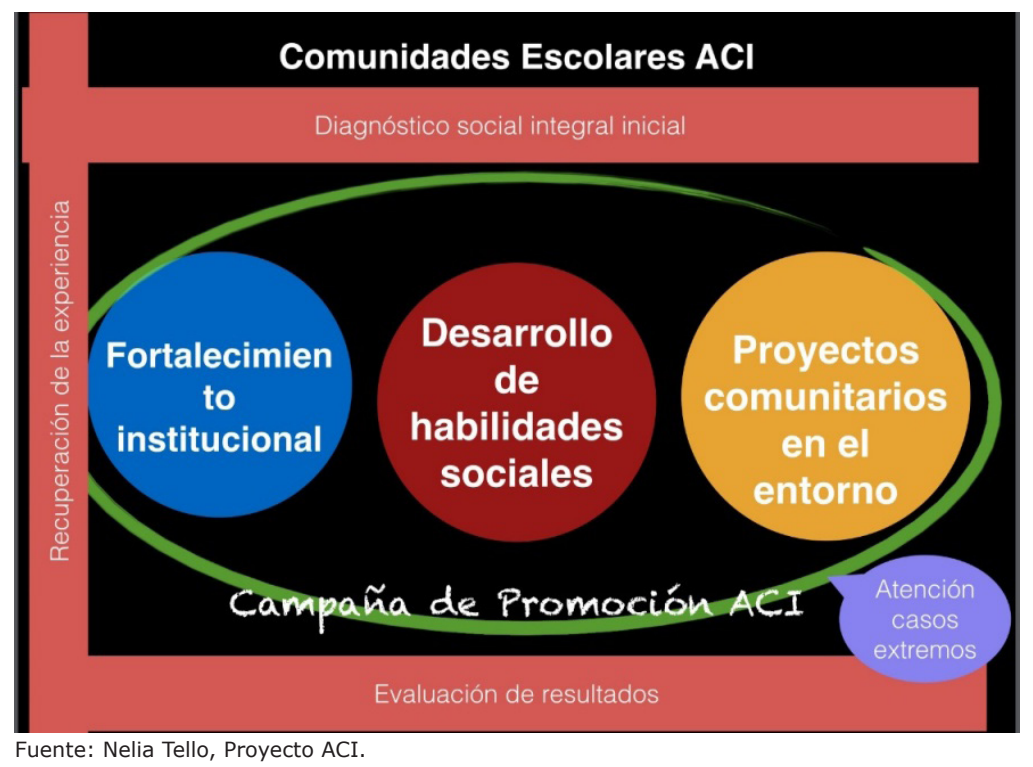

escolar desde la perspectiva social o relacional. Ello con el propósito de reflexionar en torno a la implicación de estos actores en el problema y los cambios que se requieren para modificar el clima escolar; en este sentido se trabaja el ejercicio de la autoridad asertiva y la deconstrucción de la idea de control, para substituirlo por el de disciplina, para lo cual se promueve el establecimiento de reglamentos operativos, que contengan normas, compromisos y consecuencias claras y consistentes, conocidas y aceptadas por todos los actores de la comunidad escolar.

2. El desarrollo de habilidades sociales, se refiere al trabajo que se realiza con cada uno de los actores de la comunidad escolar a través de talleres en los que se re-conozcan los procesos sociales en los que están inmersos, se asuma la corresponsabilidad que se tiene en la situación-problema y se busquen alternativas para modificar las formas de relacionarse, promoviendo la escucha activa, la empatía, el diálogo y la negociación, a fin de generar aceptación, confianza e inclusión entre los diferentes actores de la comunidad escolar. En estos talleres se trabajan temáticas básicas comunes, pero diferenciados por las actividades y los actores a los que será impartido.

3. Los proyectos comunitarios en el entorno tienen como propósito trascender lo aprendido en la escuela al llevarlo al contexto circundante, a través de la participación de algunos de los estudiantes como promotores sociales, con el propósito de desarrollar en ellos habilidades para organizar y promover acciones de interés comunitario, en donde participen los actores de la comunidad escolar y del entorno inmediato en el que se ubica la institución escolar, para facilitar así la creación de un vínculo escuelasociedad. Con ello, se estaría logrando llevar los aprendizajes que propician una convivencia solidaria fuera de la institución escolar, buscando generar redes de apoyo comunitario.

4. La atención de casos, consiste en la selección de jóvenes estudiantes que presenten problemas extremos de rechazo, desconfianza y exclusión social para trabajar con ellos habilidades sociales ACI, empleando la metodología de trabajo social de casos, que plantea no solo abordar al sujeto identificado, 
sino también a su entorno relacional, pues es ahí en donde se genera la situación-problema.

\section{Acciones transversales:}

- Diagnóstico social integral: el proceso inicia con un diagnóstico social integral sobre las formas de convivencia, que incluye algunas variables directas sobre la violencia escolar, y también variables indirectas sobre el rechazo, la desconfianza y la exclusión.

- Campaña de promoción: durante todo el tiempo de la intervención, se realiza una campaña de sensibilización permanente que contempla estrategias de reforzamiento, vivenciales y de difusión. Por medio de esta se busca sensibilizar e involucrar a otros actores y desencadenar procesos colectivos de reflexión o acción, incluyendo acciones que permiten que toda la comunidad escolar conozca los resultados de la intervención.

- Recuperación de la experiencia: el proceso de intervención se documenta de principio a fin, a través de crónicas que dan cuenta de lo sucedido en cada sesión, con cada grupo; de igual forma, se va conformando la memoria fotográfica y la testimonial que consiste en la recuperación de todos los materiales utilizados y producidos durante la intervención, que después serán analizados.

- Evaluación de resultados: esta se realiza en dos momentos de la intervención, a la mitad del proceso y al finalizarlo. La primera tiene la intención de reconocer los avances logrados y los obstáculos enfrentados para, en su caso, re-direccionar algunas de las acciones planeadas; la evaluación final tiene como propósito reconocer los resultados obtenidos y sobretodo, identificar los cambios logrados a través de la estrategia de intervención implementada.

\section{Momentos del cambio}

Intervenir en lo social supone modificar las relaciones existentes que han provocado el deterioro, fragmentación o ruptura del tejido social; en este sentido se ha desarrollado una propuesta para el diseño de modelos de intervención, cuyos elementos básicos son:

1. Definición del objeto de intervención

2. Diagnóstico integral

3. Construcción conceptual del cambio

4. Estructura metodológica

5. Evaluación y validación (Tello y Ornelas, 2015)

En una breve reseña de la propuesta, se puede decir que se inicia con la definición de la situación-problema a intervenir, de la cual se construye un diagnóstico integral y con base en los resultados de este, el trabajador social propone el cambio a lograr, lo conceptualiza, pensando en generar una realidad diferente, en la cual se propicie la aparición de nuevas relaciones que generen procesos relacionales y sociales tendientes a fortalecer la comunidad escolar, la convivencia solidaria.

Lo significativo de las intervenciones es que se generen cambios en las situaciones que se consideran conflictivas; diseñar el cambio requiere visualizar el horizonte, que en términos de Gadamer (2002) significa el "rango de visión que incluye todo cuanto puede ser visto e imaginado desde una posición, y desde donde se evalúa y comprende todo aquello que cae en dicho rango, por ello, si nos movemos se modifica nuestro horizonte; no somos prisioneros, por lo tanto, de horizontes, estos no son cerrados".

Cuando se hace referencia a la construcción de comunidades escolares de convivencia solidaria se está haciendo énfasis en el cómo se desencadenan procesos, que desde el otro, permiten 
vivir lo colectivo como un proceso comunitario. En el entendido de que lo común deviene de la interacción, del diálogo, del hacer en un espacio compartido, en este caso la escuela.

Para Arendt, el diálogo y la acción colectiva son la manera de trascender, la manera de modificar. En el espacio compartido se construye el yo con el otro, el yo implica una dimensión intersujetiva. Las relaciones, los lazos y los vínculos sociales entre todos: adultos, menores; funcionarios, maestros y padres de familia, entretejen en todas las direcciones una trama que da lugar al nosotros que nos permite ser. Como dice Octavio Paz (1960), "nunca la vida es nuestra, es de los otros, la vida no es de nadie, todos somos la vida... para que pueda ser he de ser otro, los otros que no son, si yo no existo, los otros que me dan plena existencia, no soy, no hay yo, siempre somos nosotros".

Evidentemente, este nosotros constituido por las individualidades, es producto de la incompletud que padecemos como seres humanos, pero ya no importa, porque "desde esta mirada el conocimiento implica interacción, relación, transformación mutua, co-dependencia y co-evolución" (Najmanovich). Cuando todo esto queda claro como miembros de una colectividad, lo social (la relación con el otro) se re-significa, ya que se comprende que no se puede ser sin el otro.

En este sentido, la comunidad escolar solidaria se reconoce como el nosotros, subjetivo, colectivo, que se construye y modifica en el diálogo y la acción común y al comprender que se es en lo relacional, con el otro, se aprende que se requiere de una serie de habilidades que hasta entonces tenían un significado diferente para nosotros. Cuando una comunidad escolar apertura su mirada y comprende que el crecimiento y el conocimiento dependen de lo colectivo y no de un orden dado, las habilidades básicas que se requieren para funcionar son diferentes. La primera, como dice Juliana González es estar despierta, hay que ir por el mundo con los ojos abiertos, mirar al otro. Hay que aceptarlo, en su diferencia, en sus modos, en sus maneras.

Así entendida la convivencia solidaria, hay que trabajar en la construcción social, entendiendo que la trama social la entretejen todos los seres humanos, tanto aquellos con quienes se tiene un trato cotidiano, como aquellos con los que nunca se dará un encuentro cara a cara. Lo importante es que todos los miembros de la comunidad escolar se sientan parte de ella y se asuman como responsables de lo que acontece.

Desde una mirada de la complejidad, el cambio tiene que gestarse desde una reconceptualización de la situación que se vive, que lleve a una re-significación de relación y que termine en la re-creación de espacios sociales, como se señala a continuación:

Re-conceptualización del problema: el propósito de este primer momento es que todos los actores de la comunidad escolar reconozcan el problema relacional existente; que expresen su concepción sobre los procesos que lo generan, que lo clarifiquen y a partir de ello, re-posicionen su mirada en torno al origen del problema y el involucramiento de cada actor en este.

Re-significación de las relaciones: se trata de que, una vez re-significado el problema, se lleven a cabo interacciones diferentes entre los actores, modificando aquellas que habían impedido una adecuada convivencia.

Re-creación de espacios sociales: en este momento lo que se busca es que los cambios relacionales que se han promovido al interior de las instituciones educativas, se extrapolen al resto de relaciones sociales, fuera de la escuela, es decir, con los amigos, el vecindario y el resto de la familia. 
La intervención del trabajo social en la reconstrucción del tejido social en las escuelas, desde esta propuesta, tenderá a:

Recuperar el espacio escolar como un espacio seguro, de construcción de confianza, mediado por el diálogo entre actores.

La construcción de sujetos autónomos que saben relacionarse con los otros, que se construyen en la relación con los otros, sin violencia, discriminación, desconfianza, factores que han ido desgastando y fragmentando el denominado tejido social.

La reconstrucción del sentido del nosotros, que implica el establecimiento de relaciones de solidaridad, de cooperación, a través de la reconstrucción de la memoria compartida, de encontrar la satisfacción en el bien común y la capacidad y deseo de estar juntos.

Lograr la autorregulación colectiva para que no sea uno solo de los actores el que imponga las reglas, sino que sea el colectivo quien decida cuál es la mejor forma de convivir en el espacio escolar; promoviendo que todos los integrantes cumplan los acuerdos y se sancione a quienes no lo hacen, de común acuerdo. Ello solo es posible cuando los actores de la institución educativa encuentren sentido a las reglas, comprendan por qué establecerlas y seguirlas y conozcan y asuman las consecuencias de su incumplimiento. Aprender a establecer límites para regular la convivencia, además, dotarán de estructura a los sujetos, y con ello se logrará una convivencia diferente a la dominante.

Promover la resolución de conflictos a partir del diálogo entre actores, la clarificación del problema y la corresponsabilidad en este; reconociendo el conflicto como un momento de aprendizaje para mejorar las relaciones, los vínculos.
Trabajar a partir de la diferencia de los sujetos, pero en la convergencia de ideas, de la toma de acuerdos colectivos, de beneficio común.

Fortalecer el sentido de pertenencia, para que los diferentes actores se sientan pertenecientes a la escuela, en la medida en que sus opiniones sean tomadas en cuenta en la toma de decisiones que involucra al colectivo, en la medida en que se respeten sus formas organizativas y de participación y no se pretenda que su sola presencia y escucha pasiva es participar.

Para ello, es preciso vivenciar la escuela como un espacio para la construcción de lo colectivo, no solo de la idea, sino a través de la experiencia de ser y estar en colectivo: "La fusión que requiere el entendimiento mutuo solo puede provenir de la experiencia compartida; y compartir la experiencia es inconcebible si antes no se comparte el espacio" (Bauman, 2005:38). Entonces, es necesario reconocer que si en el problema relacional todos los actores están involucrados, lo mismo pasa en su modificación, en donde el cambio solo será posible si todos los actores se involucran y hacen lo propio para lograrlo; esto solo es posible cuando se asume que el problema no es de otros, sino del nosotros y que este no existe por encima de los sujetos, sino que se entreteje en sus relaciones cotidianas.

\section{CONCLUSIONES}

Se reitera la necesidad de centrar la especificidad del trabajo social y de su intervención en lo social y construir situaciones-problema desde la mirada disciplinar. El reto del trabajo social escolar es recuperar la posibilidad de un ejercicio profesional que incida intencionalmente en los cambios sociales que tiendan a la construcción de sujetos sociales responsables y comunidades escolares en las que prive la convivencia solidaria, alejándose de la mera función administrativa y de control que se le ha asignado en este ámbito. 
El trabajo social habrá de intervenir en la reconstrucción o fortalecimiento del tejido social a través de desencadenar procesos de aceptación de la diferencia, confianza en el otro, del sentido de pertenencia a diversos colectivos, de tal manera que la participación de cada sujeto sea importante, pues "Al permitir que prospere la diversidad (de personas, actividades y credos) el espacio público posibilita la integración (o la reintegración) sin destruir las diferencias; en realidad las celebra" (Bauman, 2005:57).

Parece imprescindible que se tenga presente que la fragmentación y ruptura del tejido social es un problema estructural, no individual, pero que ello no quiere decir que no se pueda modificar con acciones en el sentido inverso, es decir desde los microespacios, en las relaciones cotidianas $y$, en este sentido, reivindicar a la escuela como espacio de posibilidad para modificar las relaciones sociales dominantes; que se asuma como un espacio de construcción de convivencia solidaria que podría trascender a otros espacios sociales; reconociendo que la escuela y los procesos educativos que en ella se gestan, juegan un papel de suma importancia en la construcción, reconstrucción y mantenimiento del tejido social, ese que nos hace estar juntos.

Resulta necesario que las propuestas para incidir en problemáticas como las expuestas, consideren a todos los actores sociales involucrados, pues centrarse en solo uno de ellos (por lo general los estudiantes), no permitirá un cambio relacional que signifique formas diferentes de convivencia.

\section{REFERENCIAS}

Ampudia, A. Santaella, G. y Eguía, S. (2016). El escenario de la violencia escolar y sus principales actores. En: Violencia escolar, aportes para la comprensión de su complejidad. SUIVEUNAM. México.

Bauman, Z. (2005). Confianza y temor en la ciudad. Vivir con extranjeros. Arcadia. Barcelona, España.

Castro, E. (2011). Reconstrucción del tejido social en el aula. Fundación Universitaria "Juan de Castellanos". Colombia. Recuperado de: http://www. revistasjdc.com

Castro, N. y Gachón, A. (2001). Tejido social y construcción de sociedad. ONG Cordillera. Centro de Estudios Municipales. Colombia. Recuperado de: www.sociedad.cl./acción/portada/ pagina.asp.
Falla, U. y Chávez, Y. (2004). Realidades y falacias de la reconstrucción del tejido social en población desplazada. Colombia. Recuperado de: http://www. redalyc.org/articulo.oa?id $=39600210$

Freire, P. (2012). Pedagogía de la autonomía. Saberes necesarios para la práctica educativa. Siglo XXI editores. México.

Giddens, A. (2001). Consecuencias de la modernidad. Alianza Editorial. México. Habermas. Teoría de la comunicación (2000). Promotores editores. Madrid, España.

Krauskopf, D. (2001). El rol de la educación. Argentina. Recuperado de: / tejido\%20social\%20en\%20el\%20aula. pdf 
Mota, L. y Sandoval, E. (2011). Acción social solidaria, confianza y diversidad cultural en América Latina (p.23-52) En: Barba, Carlos y Cohen, Ernesto (coords.). Perspectivas críticas sobre la cohesión social. CLACSO-CROP. Buenos Aires, Argentina. Recuperado de: http:// bibliotecavirtual.clacso.org.ar/ar/libros/ clacso/crop/BarbaSolano-Cohen.pdf

Najmanovich, D. (s/f). El sujeto encarnado, límites, devenir e incompletud. Recuperado de: http:// www.fac.org.ar/fec/foros/cardtran/ gral/sujeto\%20encarnado.htm

Ornelas, A. (2013). Aprender a enseñar: un acercamiento a partir de las relaciones en el aula. Tesis de maestría. UNAM-FFyL. México

Ortega, J. (2002). La escuela como ámbito de convivencia. Psicothema. México.

Paz, O. (1960). Piedra de sol. En: Poemario Libertad bajo palabra. FCE. México.

Pérez, J. (2015). Un acercamiento al clima y convivencia escolar en primarias y secundarias del Distrito Federal. Conferencia magistral en el Seminario Universitario Interdisciplinario sobre Violencia Escolar (SUIVE). UNAM. México.

Sánchez, J. A. (2011). Programas de estudio 2011. Guía para el maestro. Educación bàsica. Formación Cívica y Ética. D.F. México: Dirección General de Desarrollo Curricular (DGDC) SEP. México.
Secretaría de Seguridad Pública (2011). El Tejido Social y su fortalecimiento. Gobierno FederalSSP. México. Recuperado de: http:// www.ssp.gob.mx/portalWebApp/ ShowBinary? nodeId =/BEA\% 20 Repository/1214181//archivo

Tello, N. (2008). Apuntes de Trabajo Social. EOPSAC. México

Tello, N. (2015). Jóvenes y volencia. ENTS-UNAM-EOPSAC. México.

Tello, N. y Ornelas, A. (2015). Estrategias y modelos de intervención de trabajo social. EOPSAC-ENTS-UNAM. México.

Tello, N. y Ornelas, A. (2016). La violencia escolar, algo más que golpes e insultos entre buenos y malos: Un acercamiento desde lo social. SUIVEUNAM. México (en impresión)

Tronco del, J. (2015). La violencia escolar como problema público: Instrumentos de política para su prevención. Conferencia. Seminario Universitario Interdisciplinario sobre Violencia Escolar (SUIVE)- UNAM. México.

Valentín y Otero. Convivencia escolar: problemas y soluciones. Revista Complutense de Educación. No. 1, 12, 295-328. España.

Vázquez, J. (2008). Autoridad moral y autonomía. Una relectura del pensamiento sociológico de Ëmile Durkheim. Universidad Iberoamericana. Colección Teoría Social. México. 